\title{
On the Characterization and Enumeration of Some Generalized Trapezoidal Numbers
}

\author{
Somphong Jitman and Chakrit Phongthai \\ Department of Mathematics, Faculty of Science, Silpakorn University, Nakhon Pathom 73000, Thailand \\ Correspondence should be addressed to Somphong Jitman; sjitman@gmail.com
}

Received 4 May 2017; Accepted 16 July 2017; Published 22 August 2017

Academic Editor: Susana Montes

Copyright (c) 2017 Somphong Jitman and Chakrit Phongthai. This is an open access article distributed under the Creative Commons Attribution License, which permits unrestricted use, distribution, and reproduction in any medium, provided the original work is properly cited.

\begin{abstract}
A trapezoidal number, a sum of at least two consecutive positive integers, is a figurate number that can be represented by points rearranged in the plane as a trapezoid. Such numbers have been of interest and extensively studied. In this paper, a generalization of trapezoidal numbers has been introduced. For each positive integer $m$, a positive integer $N$ is called an $m$-trapezoidal number if $N$ can be written as an arithmetic series of at least 2 terms with common difference $m$. Properties of $m$-trapezoidal numbers have been studied together with their trapezoidal representations. In the special case where $m=2$, the characterization and enumeration of such numbers have been given as well as illustrative examples. Precisely, for a fixed 2-trapezoidal number $N$, the ways and the number of ways to write $N$ as an arithmetic series with common difference 2 have been determined. Some remarks on 3-trapezoidal numbers have been provided as well.
\end{abstract}

\section{Introduction}

A triangular number is a figurate number that can be represented by an equilateral triangular arrangement of points equally spaced. For each positive integer $\ell$, the $\ell$ th triangular number is the number of points composing a triangle with $\ell$ points on a side and is equal to the sum of the $\ell$ natural numbers of the form $\operatorname{Tri}(\ell)=1+2+3+\cdots+\ell$. The $\ell$ th triangular number can be represented as points in an equilateral triangle as in Figure 1.

Triangular numbers have been studied since the ancient Greeks. The Pythagoreans revered the Tetractys which is Tri(4). Triangular numbers have applications to other areas of number theory, such as perfect numbers and binomial coefficients. They are also practically the simplest example of an arithmetic sequence. Therefore, the triangular numbers have fascinated people and cultures all over the world (see [1$3]$ and references therein).

A trapezoidal number (see [4], e.g.) is a generalization of a triangular number defined to be a sum of at least two consecutive positive integers. Precisely, a positive integer $N$ is a trapezoidal number if

$$
N=(k+1)+(k+2)+\cdots+(k+\ell)
$$

for some integers $k \geq 0$ and $\ell \geq 2$. Trapezoidal numbers form an important class of figurate numbers that has extensively been studied (see [2-7]).

From the definition, it is not difficult to see that every trapezoidal number $N$ can be represented by a rearrangement of $N$ points in the plane as a trapezoid as in Figure 2. For convenience, denote by $T(\ell, k)$ the number of the form (1). The characterization and enumeration of trapezoidal numbers have been given in $[4,8]$. The main results are summarized as follows.

Theorem 1 ([4, Proposition 1]). Let $N$ be a positive integer. Then $N$ is a trapezoidal number if and only if $N$ is not of the form $2^{i}$ for all $i \in \mathbb{N} \cup\{0\}$.

Theorem 2 ([4, Proposition 2]). Let $N=2^{r} p_{1}^{r_{1}} p_{2}^{r_{2}} \cdots p_{s}^{r_{s}}$ be a positive integer such that $s \geq 1$ and $r \geq 0$ are integers, $p_{1}, p_{2}, \ldots, p_{s}$ are distinct odd primes, and $r_{i} \geq 1$ is an integer for all $i=1,2, \ldots, s$. Then $N$ is a trapezoidal number and there are

$$
\tau\left(\frac{n}{2^{r}}\right)-1=\left(r_{1}+1\right)\left(r_{2}+1\right) \cdots\left(r_{s}+1\right)-1
$$

ways of writing $N$ as a sum of at least two consecutive integers. 


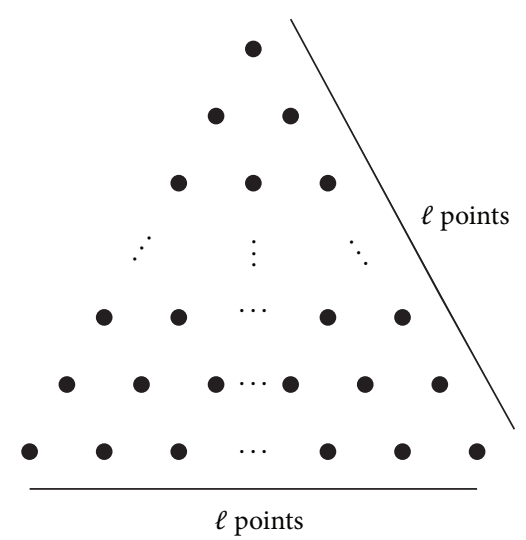

FIgURE 1: The $\ell$ th triangular number.

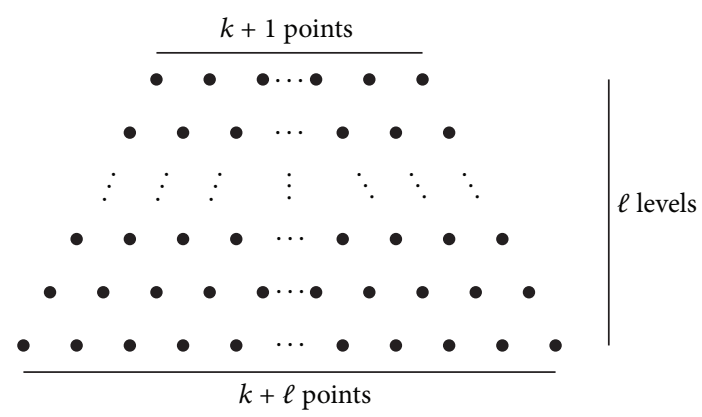

Figure 2: Trapezoidal number $T(\ell, k)$.

Some properties of nontrapezoidal numbers can be found in [9].

Triangular numbers and trapezoidal numbers have a closed connection (see Section 2 for more details) with a rectangular number which is defined to be

$$
R(\ell, k):=\ell k
$$

where $\ell \geq 2$ and $k \geq 2$ are integers. A rectangular number can be represented as a rectangle as in Figure 3.

In this paper, we focus on a general concept of trapezoidal numbers. For each positive integer $m$, a positive integer $N$ is called an $m$-trapezoidal number if $N$ can be written as an arithmetic series of at least 2 terms with common difference $m$. It follows that an $m$-trapezoidal number can be represented as

$$
\begin{aligned}
& (k+1)+(k+1+m)+(k+1+2 m)+\cdots \\
& +(k+1+m(\ell-1))
\end{aligned}
$$

for some integers $k \geq 0$ and $\ell \geq 2$. It is not difficult to see that a 1-trapezoidal number is a classical trapezoidal number. For convenience, denote by $T(\ell, k, m)$ the series in (4).

We note that an $m$-trapezoidal number is not uniquely determined by a triple $(\ell, k, m)$ (see Example 3$)$. Every $m$ trapezoidal number can be represented by an arrangement of points in the plane as a trapezoid. Some examples are given as follows.

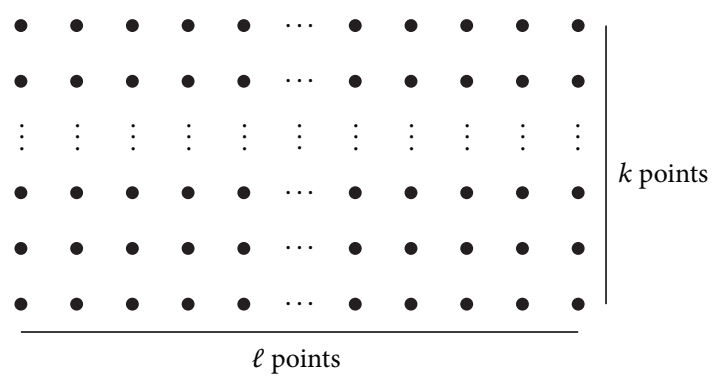

Figure 3: Rectangular number $R(\ell, k)$.

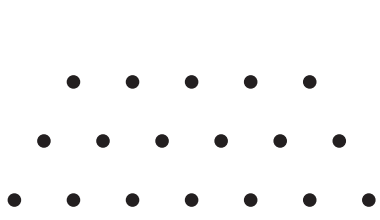

(a) $T(3,4,1)=18$

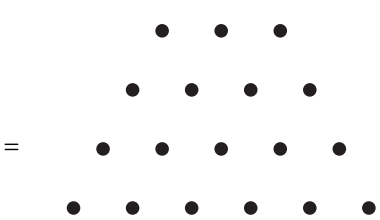

(b) $T(4,3,1)=18$
FIgURE 4: $T(3,4,1)=18=T(4,3,1)$.

Example 3. The positive integer 18 is a (1-)trapezoidal number represented in the forms of series

$$
\begin{aligned}
T(3,4,1) & =5+6+7=18=3+4+5+6 \\
& =T(4,3,1) .
\end{aligned}
$$

The above series can be represented as trapezoids of 18 points in the plane as in Figure 4.

Example 4. The numbers $T(3,4,2)=5+7+9=21$ and $T(3,4,3)=5+8+11=24$ are examples of 2 -trapezoidal and 3 -trapezoidal numbers, respectively. They can be represented as trapezoids in Figure 5.

In this paper, we focus on properties of $m$-trapezoidal numbers and their representations as trapezoids in the plane. The characterization and enumeration of $m$-trapezoidal numbers are studied in the special case where $m=2$. The paper is organized as follows. In Section 2, general properties of $m$-trapezoidal numbers are discussed as well as links with other figurate numbers. In Section 3, the characterization and enumeration of 2-trapezoidal numbers have been given together with some illustrative examples. Remarks on 3trapezoidal numbers have been provided in Section 4. Conclusion and open problems are given in Section 5.

\section{Generalized Trapezoidal Numbers}

In this section, we focus on general properties of $m$ trapezoidal numbers and links with other figurate numbers such as triangular numbers, trapezoidal number, and rectangular numbers.

First, we simplify the formula for an $m$-trapezoidal number.

Lemma 5. Let $k \geq 0, m \geq 1$, and $l \geq 2$ be integers. Then

$$
T(\ell, k, m)=\frac{\ell(2(k+1)+m(\ell-1))}{2} .
$$




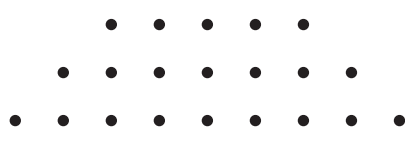

(a) $T(3,4,2)=21$

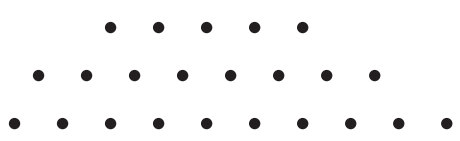

(b) $T(3,4,3)=24$

Figure 5: $T(3,4,2)=21$ and $T(3,4,3)=24$.

Proof. From the definition, we have

$$
\begin{aligned}
T(\ell, k, m)= & (k+1)+(k+1)+\cdots+(k+1) \\
& +m(1+2+\cdots+(\ell-1)) \\
= & \ell(k+1)+\frac{m(\ell-1) \ell}{2} \\
= & \frac{\ell(2(k+1)+m(\ell-1))}{2}
\end{aligned}
$$

as desired.

From the formula in Lemma 5, the following properties can be deduced.

Corollary 6. Let $m$ be a positive integer. If $m$ is even, then an $m$-trapezoidal number $T(\ell, k, m)$ is a rectangular number for all integers $k \geq 0$ and $\ell \geq 2$.

Proof. Assume that $m$ is even. Let $k \geq 0$ and $\ell \geq 2$ be integers. Then

$$
\begin{aligned}
T(\ell, k, m) & =\frac{\ell(2(k+1)+2 a(\ell-1))}{2} \\
& =\ell(k+1+a(\ell-1)) .
\end{aligned}
$$

Since $\ell \geq 2$ and $k+1+a(\ell-1) \geq 1+a \geq 2, T(\ell, k, m)$ is a rectangular number.

Corollary 7. Let $m$ be a positive integer. If $m$ is odd, then an $m$-trapezoidal number $T(\ell, k, m)$ is a rectangular number for all integers $k \geq 0$ and $\ell \geq 3$.

Proof. Assume that $m$ is odd. Let $k \geq 0$ and $\ell \geq 3$ be integers. We consider the following two cases.

Case 1 ( $\ell$ is even). Then $\ell=2 a$ for some $a \in \mathbb{N} \backslash\{1\}$ and

$$
\begin{aligned}
T(\ell, k, m) & =\frac{\ell(2(k+1)+m(\ell-1))}{2} \\
& =\frac{(2 a)(2(k+1)+m(2 a-1))}{2} \\
& =a(2(k+1)+m(2 a-1)) .
\end{aligned}
$$

Since $a \geq 2$ and $(2(k+1)+m(2 a-1)) \geq 5 \geq 2, T(\ell, k, m)$ is a rectangular number.
Case 2 ( $\ell$ is odd). Then $\ell=2 b+1$ for some $b \in \mathbb{N}$ and

$$
\begin{aligned}
T(\ell, k, m) & =\frac{\ell(2(k+1)+m(\ell-1))}{2} \\
& =\frac{(2 b+1)(2(k+1)+m(2 b+1-1))}{2} \\
& =(2 b+1)((k+1)+m b) .
\end{aligned}
$$

Since $(2 b+1) \geq 3 \geq 2$ and $((k+1)+m b) \geq 2, T(\ell, k, m)$ is a rectangular number.

From the two cases, $T(\ell, k, m)$ is rectangular for all $k \geq 0$ and $\ell \geq 3$.

Trapezoidal numbers, $m$-trapezoidal numbers, rectangular numbers, and triangular numbers are linked via the following relations.

Theorem 8. Let $\ell, k, m$ be integers such that $\ell \geq 2, m \geq 1$, and $k \geq 0$. Then

$$
T(\ell, k, m)=T(\ell, k, m-i)+(m-i) \operatorname{Tri}(\ell-1)
$$

for all positive integers $i \leq m$.

Proof. Let $i$ be a positive integer. Then

$$
\begin{aligned}
T(\ell, k, m)= & \frac{\ell(2(k+1)+m(\ell-1))}{2} \\
= & \frac{\ell(2(k+1)+i \ell-i+i+m \ell-m-i \ell)}{2} \\
= & \frac{\ell(2(k+1)+i(\ell+1))}{2} \\
& +\frac{\ell(m \ell-m+i-i \ell)}{2} \\
= & \frac{\ell(2(k+1)+i(\ell+1))}{2} \\
& +\frac{(m-i)(\ell-1) \ell}{2} \\
= & T(\ell, k, i)+(m-i) \operatorname{Tri}(\ell-1) .
\end{aligned}
$$

Hence, the result follows.

The next corollary follows immediately from Theorem 8.

Corollary 9. Let $\ell, k, m$ be integers such that $\ell \geq 2, m \geq 1$, and $k \geq 0$. Then the following statements hold:

(1) $T(\ell, k, m)=T(\ell, k, 1)+(m-1) \operatorname{Tri}(\ell-1)$.

(2) $T(\ell, k, m)=T(\ell, k, m-1)+\operatorname{Tri}(\ell-1)$. 


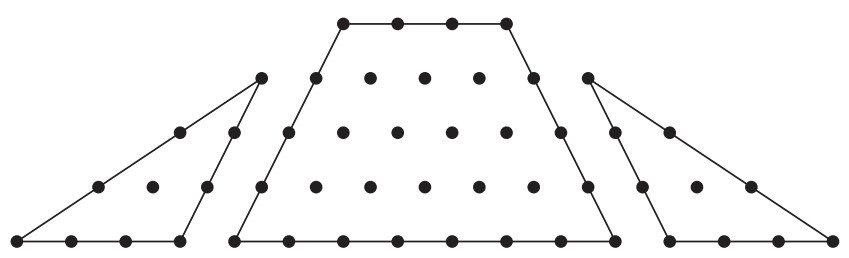

Figure 6: $T(5,3,3)=T(5,3,1)+2 \operatorname{Tri}(4)$.

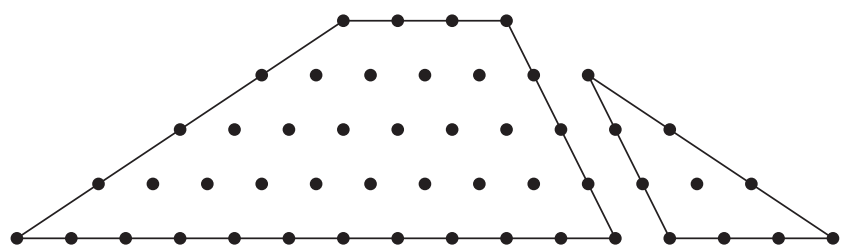

Figure 7: $T(5,3,3)=T(5,3,2)+\operatorname{Tri}(4)$.

Illustrative examples of results in Corollary 9 are given as follows.

Example 10. Let $\ell=5, \ell=5, k=3$ and $m=3$. From Theorem 8, we have

$$
\begin{aligned}
T(5,3,3) & =4+7+10+13+16 \\
& =(4+5+6+7+8)+2(1+2+3+4) \\
& =T(5,3,1)+2 \operatorname{Tri}(4), \\
T(5,3,3) & =4+7+10+13+16 \\
& =(4+6+8+10+12)+(1+2+3+4) \\
& =T(5,3,2)+\operatorname{Tri}(4) .
\end{aligned}
$$

The above relations can be represented in the plane as in Figures 6 and 7.

Theorem 11. Let $m$ and $N$ be positive integers. If $N$ is an $m$ trapezoidal number such that $N \geq m+4$, then $N$ can be written as a sum of a rectangular number and an $m$-trapezoidal number.

Proof. Assume that $N$ is an $m$-trapezoidal number such that $N \geq m+4$. Then

$$
N=\frac{b(2(a+1)+m(b-1))}{2}=T(b, a, m)
$$

for some integers $a \geq 1$ and $b \geq 2$.

Let $N_{1}=(a+1) b$ and $N_{2}=(b-1)(2 m+m(b-2)) / 2=$ $T(b-1, a, m)$. Then $N_{1}$ is a rectangular number and $N_{2}$ is an $m$-trapezoidal number. It follows that

$$
\begin{aligned}
N_{1}+N_{2} & =(a+1) b+\frac{(b-1)(2 m+m(b-2))}{2} \\
& =\frac{b(2(a+1)+(m)(b-1))}{2}=N .
\end{aligned}
$$

Hence, $N$ is a sum of a rectangular number and an $m$ trapezoidal number.

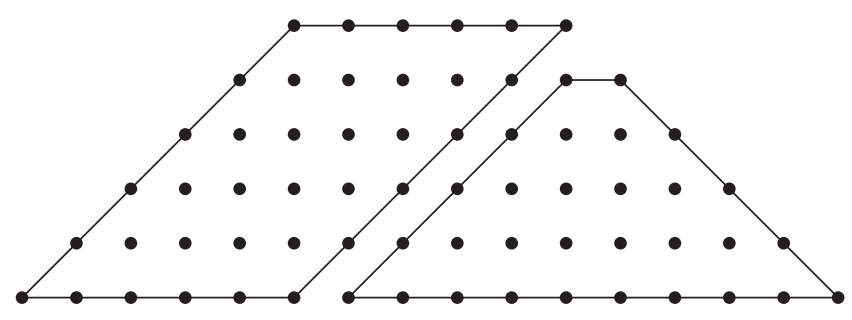

Figure 8: $T(6,5,2)=R(6,6)+T(5,1,2)$.

Example 12. Let $\ell=6, k=5$, and $m=2$. Then

$$
\begin{aligned}
T(6,5,2) & =6+8+10+12+14+16 \\
& =6 \cdot 6+(2+4+6+8+10) \\
& =R(6,6)+T(5,1,2)
\end{aligned}
$$

which can be represented in the plane as in Figure 8.

\section{Characterization and Enumeration of 2-Trapezoidal Numbers}

In this section, we focus on the special case where $m=2$. The characterization and enumeration of 2-trapezoidal numbers are given together with some illustrative examples.

The characterization of 2-trapezoidal numbers is given in the next theorem which is totally different from the case of 1 -trapezoidal numbers in Theorem 1.

Theorem 13. Let $N \geq 2$ be an integer. Then $N$ is a 2-trapezoidal number if and only if $N$ is not a prime.

Proof. Assume that $N$ is a 2-trapezoidal number. By Corollary $6, N$ is a rectangular number. Hence, $N$ is not a prime number.

Conversely, assume that $N$ is not a prime number. Then there exist integers $1<r \leq s<N$ such that $N=r s$. Choose $\ell=r$ and $k=s-r$. Then $\ell \geq 2, k \geq 0$, and

$$
\begin{aligned}
N & =r s=\ell(k+\ell)=\frac{\ell(2(k+1)+2(\ell-1))}{2} \\
& =T(\ell, k, 2) .
\end{aligned}
$$

Hence, $N$ is a 2-trapezoidal number as desired.

From the proof of Theorem 13, a 2-trapezoidal number $N$ can be represented as a series $T(\ell, k, 2)$ via the following steps:

(1) Determine the divisors $r$ of $N$ such that $1<r \leq \sqrt{N}$.

(2) For each $r$, compute $s=N / r$.

(3) Write $T(\ell, k, 2)$, where $\ell=r$ and $k=s-r$.

Let us consider the following examples.

Example 14. Consider the 2-trapezoidal number $N=54$. We have $54=2 \cdot 3^{3}$ which can be written as arithmetic series of at least 2 terms with common difference 2 as in Table 1 . 
TABLE 1: Trapezoidal representations of 54.

\begin{tabular}{lccc}
\hline$\ell=r$ & $s=N / r$ & $k=s-r$ & $T(\ell, k, 2)$ \\
\hline 2 & 27 & 25 & $26+28$ \\
3 & 18 & 15 & $16+18+20$ \\
6 & 9 & 3 & $4+6+8+10+12+14$ \\
\hline
\end{tabular}

TABLE 2: Trapezoidal representations of 100 .

\begin{tabular}{lccc}
\hline$\ell=r$ & $s=N / r$ & $k=s-r$ & $T(\ell, k, 2)$ \\
\hline 2 & 50 & 48 & $49+51$ \\
4 & 25 & 21 & $22+24+26+28$ \\
5 & 20 & 15 & $16+18+20+22+24$ \\
10 & 10 & 0 & $1+3+5+7+9+11+13+15+17+19$ \\
\hline
\end{tabular}

TABLE 3: Trapezoidal representations of 175.

\begin{tabular}{lccc}
\hline$\ell=r$ & $s=N / r$ & $k=s-r$ & $T(\ell, k, 2)$ \\
\hline 5 & 35 & 30 & $31+33+35+37+39$ \\
7 & 25 & 18 & $19+21+23+25+27+29+31$ \\
\hline
\end{tabular}

Example 15. Consider the 2-trapezoidal number $N=100$. Then $100=2^{2} \cdot 5^{2}$ which can be written as arithmetic series of at least 2 terms with common difference 2 as in Table 2.

Example 16. Consider the 2-trapezoidal number $N=175$. Then $175=5^{2} \cdot 7$ which can be written as arithmetic series of at least 2 terms with common difference 2 as in Table 3.

By the definition, every 2-trapezoidal number can be written as an arithmetic series with common difference 2 . In the following theorem, we determine the number of ways to write a 2-trapezoidal number in terms of an arithmetic series of at least 2 terms with common difference 2 .

Theorem 17. Let $N$ be a 2-trapezoidal number. Then the number of ways to write $N$ as an arithmetic series of at least 2 terms with common difference 2 is

$$
\begin{gathered}
\frac{\tau(N)-1}{2} \text { if } N \text { is a square, } \\
\frac{\tau(N)}{2}-1 \text { if } N \text { is not a square, }
\end{gathered}
$$

where $\tau(N)$ is the number of divisors of $N$.

Proof. From the proof of Theorem 13, it follows that $N=r s$ for some integers $1<r \leq s$. Next, we consider the following two cases.

Case 1 ( $N$ is a square). In this case, we have $1<r \leq s$. Then the number of ways to write $N$ as an arithmetic series of at least 2 terms with common difference 2 is the number of divisors $r$ of $N$ such that $1<r \leq \sqrt{N}$. Since $N$ is a square, $N=$ $p_{1}^{r_{1}} p_{2}^{r_{2}} \cdots p_{s}^{r_{s}}$ for some $s \geq 1, p_{1}, p_{2}, \ldots, p_{s}$ are distinct odd primes, and $r_{i}$ is an even positive integer for all $i=1,2, \ldots, s$. Then the number of divisors of $N$ is $\tau(N)=\left(r_{1}+1\right)\left(r_{2}+\right.$ $1) \cdots\left(r_{s}+1\right)$ which is odd. Hence, the number of ways to write
TABLE 4: Number of representations of square 2-trapezoidal numbers.

\begin{tabular}{ll}
\hline$N$ & $\frac{\tau(N)-1}{2}$ \\
\hline $25=5^{2}$ & $\frac{(2+1)-1}{2}=1$ \\
$36=2^{2} \cdot 3^{2}$ & $\frac{(2+1)(2+1)-1}{2}=4$ \\
$49=7^{2}$ & $\frac{(2+1)-1}{2}=1$ \\
$81=3^{4}$ & $\frac{(2+1)(2+1)-1}{2}=4$ \\
$100=2^{2} \cdot 5^{2}$ & $\frac{(8+1)-1}{2}=4$ \\
$256=2^{8}$ & $\frac{(4+1)(2+1)-1}{2}=7$ \\
$400=2^{4} \cdot 5^{2}$ &
\end{tabular}

$N$ as an arithmetic series of at least 2 terms with common difference 2 is $(\tau(N)-1) / 2+1-1=(\tau(N)-1) / 2$.

Case 2 ( $N$ is not a square). In this case, we have $1<r<s$. Then the number of ways to write $N$ as an arithmetic series of at least 2 terms with common difference 2 is the number of divisors $r$ of $N$ such that $1<r<\sqrt{N}$. Since $N$ is not a square, $N=p_{1}^{r_{1}} p_{2}^{r_{2}} \cdots p_{s}^{r_{s}}$, where $s \geq 1, p_{1}, p_{2}, \ldots, p_{s}$ are distinct odd primes and $r_{i}$ is a positive integer for all $i=$ $1,2, \ldots, s$ such that $r_{j}$ is odd for some $1 \leq j \leq s$. Then the number of divisors of $N$ is $\tau(N)=\left(r_{1}+1\right)\left(r_{2}+1\right) \cdots\left(r_{s}+1\right)$ which is even. Therefore, the number of ways to write $N$ as an arithmetic series of at least 2 terms with common difference 2 is $\tau(N) / 2-1$.

From the two cases, the result follows.

The next corollary is a direct consequence of Theorem 17.

Corollary 18. Let $N$ be a 2-trapezoidal number. Then $N$ has a unique representation as an arithmetic series of at least 2 terms with common difference 2 if and only one of the following statements holds:

(1) $N$ is a product of two distinct primes.

(2) $N$ is the square of a prime.

(3) $N$ is the cube of a prime.

Some illustrative examples of the number of ways to write a 2-trapezoidal number $N$ as an arithmetic series of at least 2 terms with common difference 2 are shown in Tables 4 and 5 .

\section{Some Properties of 3-Trapezoidal Numbers}

In this section, we focus on properties of 3-trapezoidal numbers. A necessary condition for a positive integer to be a 3-trapezoidal number is given. However, this condition is not sufficient.

Theorem 19. Let $N$ be a positive integer. If $N$ is a 3-trapezoidal number, then $N$ is not in the form of $2^{i}$ for all $i \in \mathbb{N} \cup\{0\}$. 
TABLE 5: Number of representations of nonsquare 2-trapezoidal numbers.

\begin{tabular}{lc}
\hline$N$ & $\frac{\tau(N)}{2}-1$ \\
\hline $10=2 \cdot 5$ & $\frac{(1+1)(1+1)}{2}-1=2-1=1$ \\
$24=2^{3} \cdot 3$ & $\frac{(3+1)(1+1)}{2}-1=3$ \\
$27=3^{3}$ & $\frac{(1+1)(1+1)(1+1)}{2}-1=1-1=3$ \\
$42=2 \cdot 3 \cdot 7$ & $\frac{(1+1)(3+1)}{2}-1=4-1=3$ \\
$54=2 \cdot 3^{3}$ &
\end{tabular}

Proof. Assume that $N$ is a 3-trapezoidal number. Then

$$
N=\frac{\ell(2(k+1)+3(\ell-1))}{2}
$$

for some $\ell \geq 2$ and $k \geq 0$. We consider the following two cases.

Case 1 ( $\ell$ is odd). It follows that $3(\ell-1)$ is even and $(2(k+$ $1)+3(\ell-1)) / 2 \in \mathbb{N}$. It follows that $\ell \geq 3$ is odd and $\ell \mid N$. Hence, $N \neq 2^{i}$ for all $i \in \mathbb{N} \cup\{0\}$.

Case 2 ( $\ell$ is even). We have that $3(\ell-1)$ is odd and $2(k+1)+$ $3(\ell-1)$ is odd. Since $\ell / 2 \in \mathbb{N}$, it follows that $(2(k+1)+3(\ell-1)) \mid$ $N$ and $2(k+1)+3(\ell-1) \geq 5$. Hence, $N \neq 2^{i}$ for all $i \in \mathbb{N} \cup\{0\}$.

Altogether, we have that $N \neq 2^{i}$ for all $i \in \mathbb{N} \cup\{0\}$ as desired.

We note that the necessary condition given in Theorem 19 is not sufficient. It is not difficult to see that 6 is not of the form $2^{i}$ for all $i \in \mathbb{N} \cup\{0\}$ but 6 is not a 3 -trapezoidal number.

\section{Conclusion and Remarks}

A general concept of trapezoidal numbers has been introduced. Some properties of $m$-trapezoidal numbers have been determined as well as links with other figurate numbers. Complete characterization and enumeration of 2-trapezoidal numbers are given. A necessary condition of a positive integer to be a 3-trapezoidal number is determined. However, the given condition is not sufficient.

In general, it is interesting to study the characterization and enumeration of $m$-trapezoidal numbers with $m \geq 3$.

\section{Conflicts of Interest}

The authors declare that there are no conflicts of interest regarding the publication of this paper.

\section{Acknowledgments}

This research was supported by the Thailand Research Fund and the Office of Higher Education Commission of Thailand under Research Grant MRG6080012.

\section{References}

[1] S. Asadulla, "Thirty-nine [thirty] perfect numbers and their divisors," International Journal of Mathematics and Mathematical Sciences, vol. 9, no. 1, pp. 205-206, 1986.

[2] P. J. Berana, J. Montalbo, and D. Magpantay, "On triangular and trapezoidal numbers," Asia Pacific Journal of Multidisciplinary Research, vol. 3, pp. 76-81, 2015.

[3] T. Verhoeff, "Rectangular and trapezoidal arrangements," Journal of Integer Sequences, vol. 2, Article 99.1.6 (HTML document) pages, 1999.

[4] C. Gamer, D. W. Roeder, and J. J. Watkins, "Trapezoidal Numbers," Mathematics Magazine, vol. 58, no. 2, pp. 108-110, 1985.

[5] C. Feinberg-McBrian, "The case of trapezoidal numbers," Mathematics Teacher, vol. 89, pp. 16-24, 1996.

[6] P. W. Haggard and K. L. Morales, "Discovering relationships and patterns by exploring trapezoidal numbers," International Journal of Mathematical Education in Science and Technology, vol. 24, no. 1, pp. 85-90, 1993.

[7] J. Smith, “Trapezoidal numbers," Mathematics in School, vol. 5, p. 42, 1997.

[8] R. Guy, "Sums of consecutive integers," The Fibonacci Quarterly. Official Organ of the Fibonacci Association, vol. 20, no. 1, pp. 3638, 1982.

[9] C. Jones and N. Lord, "Characterising non-trapezoidal numbers," The Mathematical Gazette, vol. 83, no. 497, pp. 262-263, 1999. 


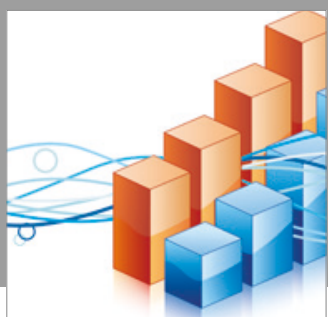

Advances in

Operations Research

vatersals

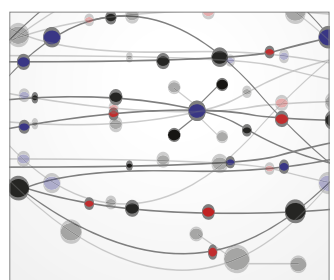

\section{The Scientific} World Journal
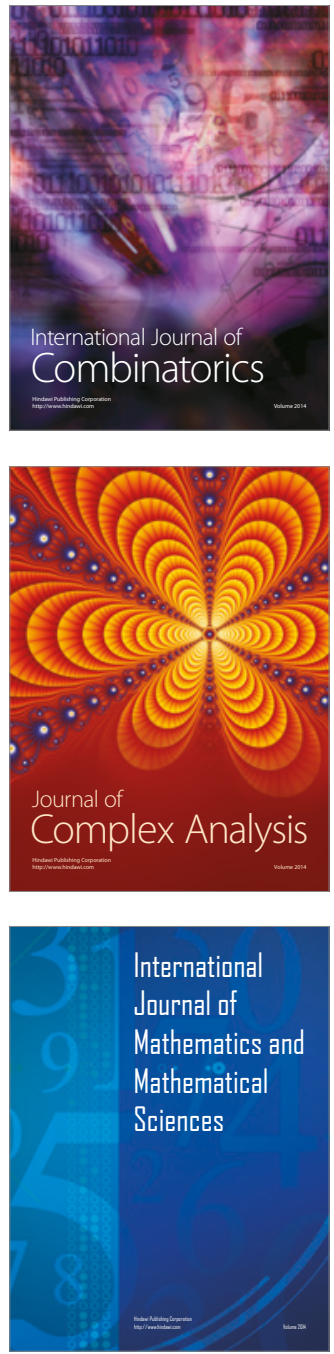
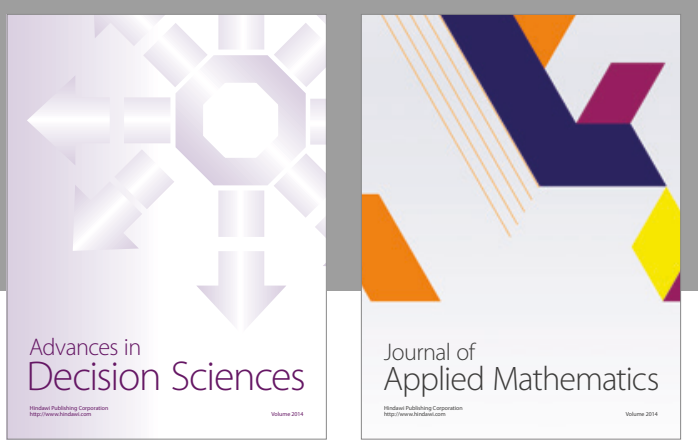

Algebra

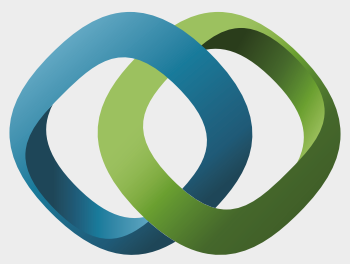

\section{Hindawi}

Submit your manuscripts at

https://www.hindawi.com
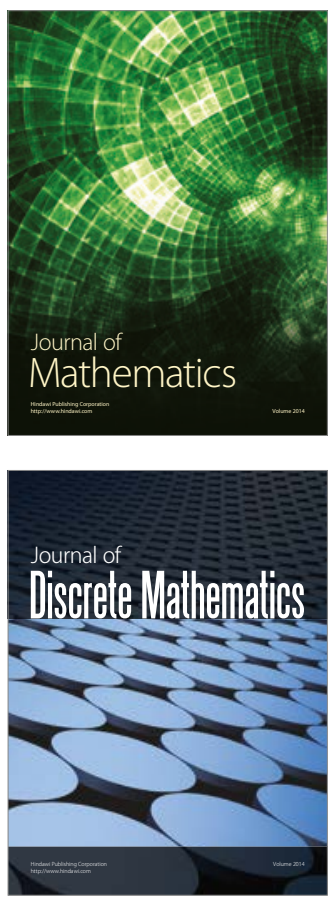

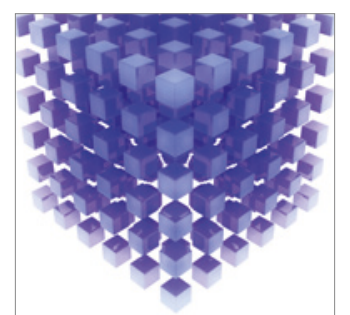

Mathematical Problems in Engineering
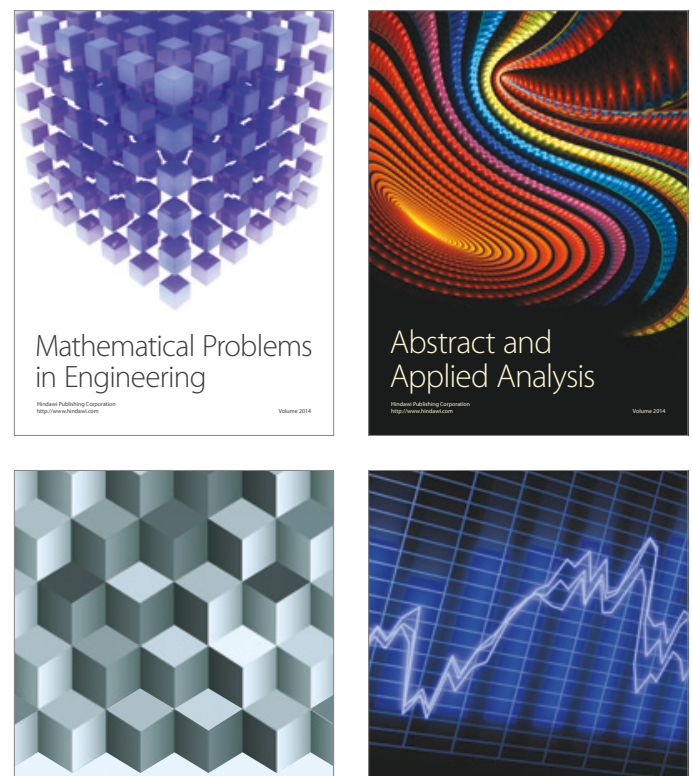

Journal of

Function Spaces

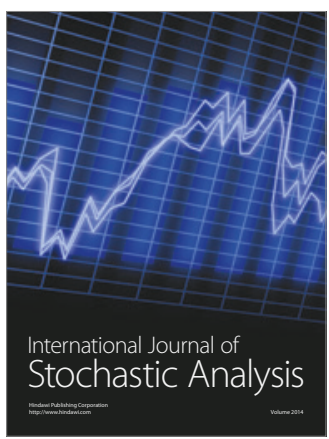

Probability and Statistics
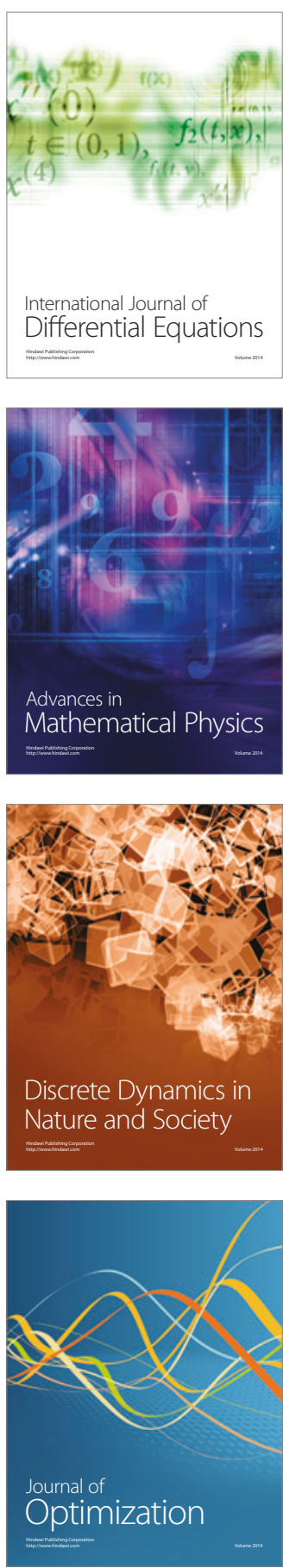